This item was submitted to Loughborough's Research Repository by the author.

Items in Figshare are protected by copyright, with all rights reserved, unless otherwise indicated.

\title{
Multifunctional control design for modular plug-and-play battery storage in DC microgrids
}

\author{
PLEASE CITE THE PUBLISHED VERSION
}

https://doi.org/10.1109/ISIE45063.2020.9152414

PUBLISHER

IEEE

VERSION

AM (Accepted Manuscript)

\section{PUBLISHER STATEMENT}

(c) 2020 IEEE. Personal use of this material is permitted. Permission from IEEE must be obtained for all other uses, in any current or future media, including reprinting/republishing this material for advertising or promotional purposes, creating new collective works, for resale or redistribution to servers or lists, or reuse of any copyrighted component of this work in other works.

\section{LICENCE}

\section{All Rights Reserved}

\section{REPOSITORY RECORD}

Li, Fulong, Haoge Xu, and Zhengyu Lin. 2020. "Multifunctional Control Design for Modular Plug-and-play Battery Storage in DC Microgrids". Loughborough University. https://hdl.handle.net/2134/12909806.v1. 


\section{Multifunctional Control Design for Modular Plug- and-play Battery Storage in DC Microgrids}

\author{
Fulong Li \\ School of Mechanical, Electrical and \\ Manufacturing Engineering \\ Loughborough University \\ Leicestershire, United Kingdom \\ F.Li@,lboro.ac.uk
}

\author{
Zhengyu Lin \\ School of Mechanical, Electrical and \\ Manufacturing Engineering \\ Loughborough University \\ Leicestershire, United Kingdom \\ Z.Lin@,1boro.ac.uk
}

\author{
Haoge $\mathrm{Xu}$ \\ School of Mechanical, Electrical and \\ Manufacturing Engineering \\ Loughborough University \\ Leicestershire, United Kingdom \\ H.Xu2@,1boro.ac.uk
}

\begin{abstract}
Plug-and-Play (P\&P) performance facilitates the modularity of DC microgrids. The realization of $P \& P$ operation relies on the control design of $\mathrm{DC}$ microgrids. Conventional control methods are normally designed for steady operation of a DC microgrid, neglecting or partially sacrifices the availability of P\&P operations. Some bottom layer's control designs such as droop control, from a hierarchical control scheme perspective for example, are inherently able to realize $P \& P$ operations. However, such methods have limitations in terms of power sharing accuracy. This paper proposes a control scheme that reconfigures hierarchical control and makes it more compatible for different P\&P operation situations in DC microgrids. In this control scheme, Automatic Mater-Slave (AMS) control is implemented in the secondary control layer to automatically respond to those cases in the absence of communication or the failure of the master module. The proposed control scheme is validated by MATLAB/Simulink simulation.
\end{abstract}

Keywords-battery storage, DC microgrids, droop control, master-slave control, plug-and-play

\section{INTRODUCTION}

Increasingly, clean renewable energy sources such as wind power and solar power are supplementing and/or replacing, fossil fuels as the power generation to electrical grids. Effective and efficient integration of such diverse energy sources, to their full potential, necessitates changes to the architecture and management of those grids. DC microgrids have been a hot research topic in the past decades. They are more flexible and compatible in integrating with those renewable energy sources than conventional AC utility grid, marked with higher efficiency, reliability and controllability [1-3]. A typical plug-and-play (P\&P) DC microgrid is shown in Fig. 1.

The requirement of $\mathrm{P} \& \mathrm{P}$ arises as the $\mathrm{DC}$ microgrids are labeled with flexibility and scalability [2], which is also needed for the commercialisation of DC microgrids. Conventional power stations usually have relative constant power generation, they thus have less requirement for the source side P\&P operation. While in DC microgrids, the input source could be various and intermittent. The diversity of input source in DC microgrids makes the whole DC system gain more controllability but also challenge. For the easy-touse DC microgrids, it is necessary to make those input sources to realize $\mathrm{P} \& \mathrm{P}$ operation just like conventional loads.

The realization of P\&P operation comes from the design of control schemes. A hierarchical control scheme is normally applied in a DC microgrids [3]. The inclusion of centralized or decentralized communication in the control scheme makes the system operate smarter and more effective. However, the absence of centralized communication could also lead the

This work has received funding from the U.K. EPSRC UKRI Innovation Fellowship scheme under grant No. EP/S001662/2, and the European Union's Horizon 2020 research and innovation programme under grant agreement No.734796.

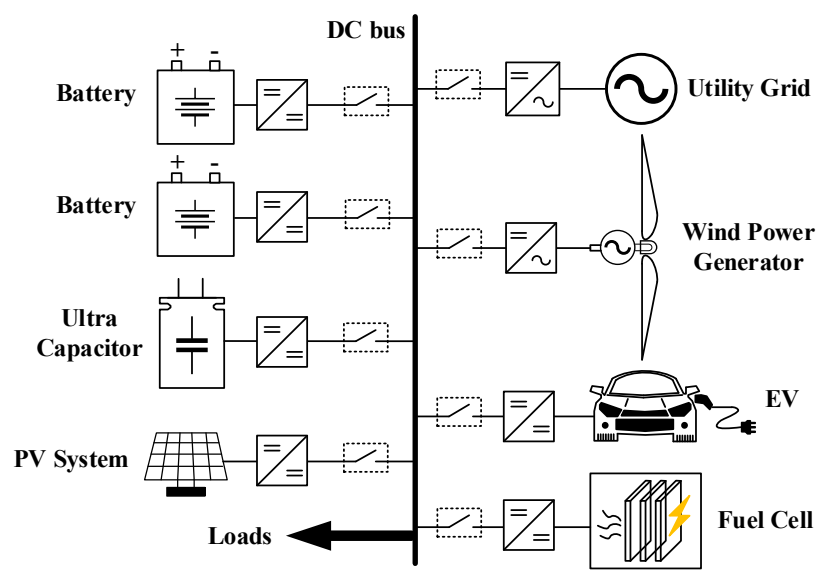

Fig. 1. A typical plug-and-play DC microgrid.

system into chaos in some cases. Therefore, it is important to enhance the performance of DC microgrids without communication.

Conventional control methods to realize $\mathrm{P} \& \mathrm{P}$ performance of input sources are droop-based [4-6] control methods. Droop control has inherent characteristic for P\&P operation. However, it is notorious for the power sharing accuracy [4]. A lot of methods have been proposed for such drawbacks. For example, a virtual negative cable resistance secondary control method in paper [6] is proposed to solve such issues. In terms of droop related P\&P research, paper [7] proposed a two degree of-freedom admittance-type droop control method to fulfill the requirements of the P\&P operation where the stability of such operation is also studied. In terms of stability study of P\&P DC microgrids, stabilizing controller design is also proposed in [8]. Paper [9] introduces the state of charge as a parameter in the droop coefficient, which is one of the commonly seen mutations of droop control, for the P\&P electrical vehicle. However, to achieve P\&P operation in droop-based control, communication is required to deal with those drawbacks otherwise, droop control is not appropriate the DC microgrid with the high requirement of power sharing accuracy.

Master slave (MS) control [10] is another control method to realize P\&P operation but only limited to the slave modules. The mechanism of MS control is that master module works under voltage control mode to maintain the DC bus voltage and slave modules work under current control mode to share the power of master module. However, once the master module is broken, then the remaining slave modules cannot maintain the DC bus voltage. Automatic master slave (AMS) control is then proposed in paper [11] to the solve this 
problem. When the master module is unavailable, the remaining slave module will automatically select a module as a new master module based on their output current. However, this paper only considers unidirectional current flow. For the battery storage in the DC microgrids, bidirectional current flow always needs to be considered. Besides, conventional diode array for current selection is also not applicable for bidirectional output current selection.

Driven by above concerns, this paper proposed a multifunctional control scheme for the realization of modular, scalable and prefabricated P\&P battery storage in the DC microgrids. A fact is that battery is mostly used to maintain the DC voltage as its bidirectional power flow and ability to adjust the system deficit and surplus power. Using battery storage to maintain the DC bus voltage can also avoid complex seamless on/off-grid transfer required gridcontrolled DC microgrids. In this control scheme, the system is no longer relied on the communication to manage the power sharing and gains more self-governance. It contains three parts, which are double loop voltage/current control, droop control and AMS control, respectively. Droop control is implemented in this control scheme to make the DC system compatible for other voltage source, such as fuel cell. AMS control is the main contributor for P\&P performance. It will automatically adjust the voltage or current controlled mode though a bidirectional current comparison algorithm. Therefore, each individual battery storage unit with such a control can be freely plugged-in and plugged-out without affecting system operation. A typical advantage is that the battery storage can maintain the bus voltage uninterruptable even considering their degradation or unpredicted issues happed in single or multiple battery storage. Compared with conventional methods, the proposed control scheme makes the battery storage in DC microgrid gain more scalability and flexibility in terms of the P\&P operation. It can also be integrated with communication to participate in the energy management or operates in self-governance without it.

The rest of this paper is organized as follows: Section II introduces the control background of P\&P performance. Section III provides detailed controller design process. The simulation validation is illustrated in Section IV and many situations are simulated. Finally, the concluding remarks are made in Section V.

\section{CONTROL BACKGROUNd OF Plug-AND-PlAy}

The availability of $P \& P$ performance is mainly based on configuration of control layer. Commonly applied control structure in DC microgrids is hierarchical control, which is shown in Fig. 2. The primary control includes voltage and current control and droop control. The secondary control contains the manipulations of DC bus voltage, etc. The tertiary control is communication-related power/energy management, which also centrally control the parameters from the primary and secondary control layers.

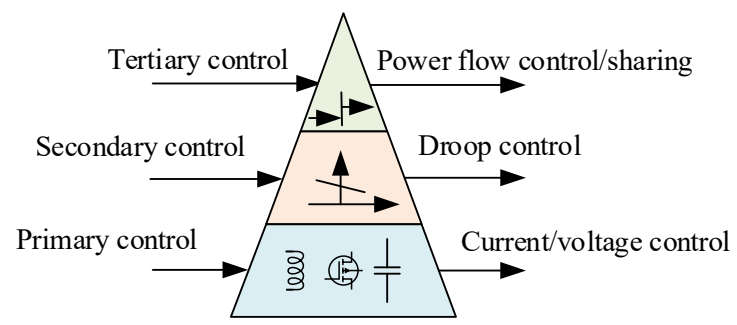

Fig. 2. A hierarchical control scheme in a DC microgrid.
Generally, P\&P performance can be realized through communications. However, the absence of communication will make the DC system move into chaos if without proper primary and secondary control design.

\section{A. Droop Control}

Droop control is one of the most widely applied control methods in DC microgrids due to its simplicity. A DC/DC converter regulated distributed source (DS) embedded with droop control can be simplified as Fig. 3.

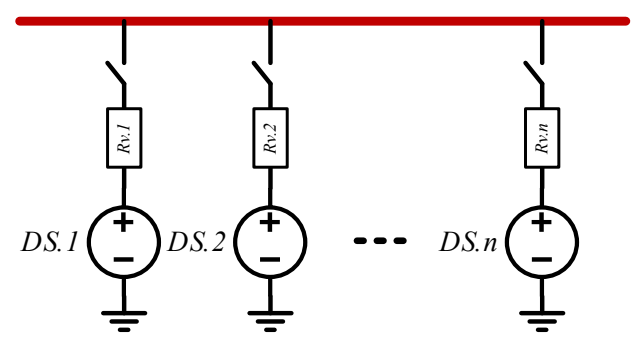

Fig. 3. Simplified droop control in P\&P DC microgrids.

The most significant characteristic of droop control is that they are paralleled voltage sources with virtual resistances. If the virtual resistances are designed same and neglecting the cable resistance, the average current sharing can be achieved between those sources. However, it is difficult to meet those conditions in practical. Apart from above conditions, the converters themselves are difficult to be designed to be identical.

\section{B. Master-Slave Control}

Different from droop control, MS control is much easier to be implemented in DC microgrids. A master module works at voltage source mode to maintain the DC bus voltage and the remaining slave modules work at current source mode, which is shown in Fig. 4. The master module can be either intergraded with droop control or not. The slave module can be freely plugged-in and plugged-out.

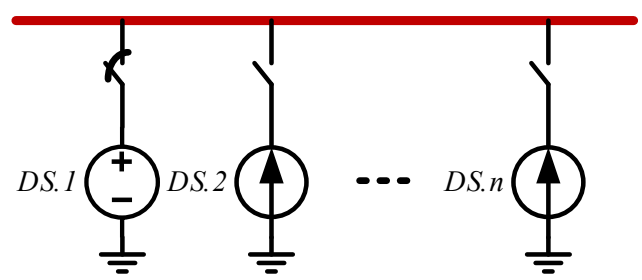

Fig. 4. Simplified MS control in P\&P DC microgrids.

This method does not have current sharing accuracy issues. However, the failure of master module can always be devastating as the remaining slave module cannot maintain the DC bus voltage. It thus suffers from single point of failure.

\section{Control Design}

In this section, the proposed control scheme will be introduced. This control scheme is still in hierarchy. This paper emphasizes the primary and secondary control to enhance the self-governance of converters. For the centralized communication, it will be explained how to integrate with proposed control.

\section{A. Modelling of Converter}

A bidirectional buck-boost converter is used in this paper. The topology of such converter is shown in Fig. 5. 


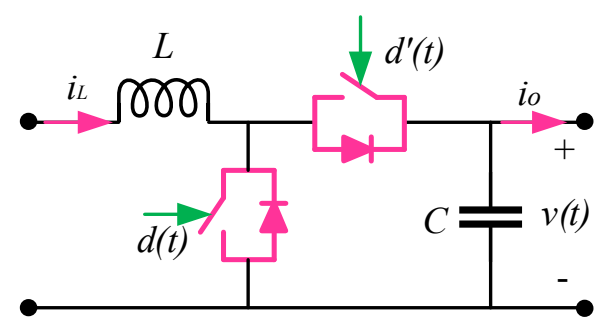

Fig. 5. The topology of bidirectional buck-boost converter. (1).

A book-keeping dynamic analysis is shown in equation

$$
\left\{\begin{array}{l}
L \frac{d i_{L}(t)}{d t}=v_{g}(t)-d^{\prime}(t) v_{o}(t) \\
C \frac{d v_{o}(t)}{d t}=d^{\prime}(t) i_{L}(t)-i_{o}(t)
\end{array}\right.
$$

where $v_{g}$ is input source voltage; $v_{o}$ is converter output voltage; $d$ is the duty cycle and $d^{\prime}$ equals to $1-d ; i_{o}$ is output current and $i_{L}$ is inductor current.

Applying small signal analysis, the converter transfer functions of control to inductors and output voltage can be easily attained [12]. Based-on those transfer function, the inner current and voltage controller can be easily design through book-keeping methods [13].

\section{B. Primary Control Design}

The primary controller includes aforementioned double loop voltage/current control and droop control.

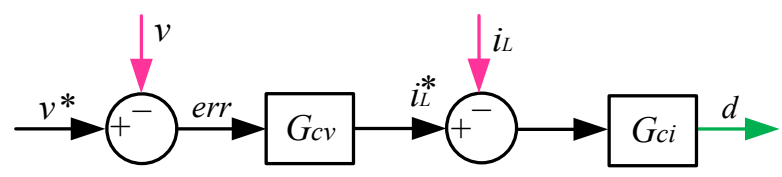

Fig. 6. Double loop control blocks of converter.

The inner loop current and outer loop voltage controller are both PI controllers, which is shown in Fig. 6. They are written in equation (2) and equation (3).

$$
\begin{gathered}
G_{c i}=G_{i m} \cdot\left(1+\frac{\omega_{z}}{s}\right) \\
G_{c v}=G_{v m} \cdot\left(1+\frac{\omega_{z v}}{s}\right)
\end{gathered}
$$

Droop controller is included in primary control layer, considering that it may have other generation functioning as voltage source, such as fuel cell.

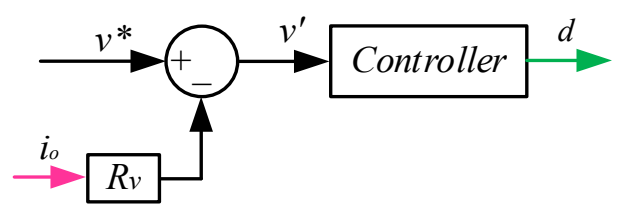

Fig. 7. Droop control blocks.

The control block of droop control is shown in Fig. 7. $v^{\prime}$ replace $v^{*}$ by introducing virtual resistance $R_{v}$ to form the new voltage reference, which can be written in equation (4).

$$
v^{\prime}=v^{*}-i_{o} R_{v}
$$

\section{Secondary Control-AMS Control}

The main idea of Automatic Mater-Slave (AMS) control is that the DC system will not be impacted by the single failure of the master module because the remaining slave modules will automatically select another master module to maintain the bus voltage.

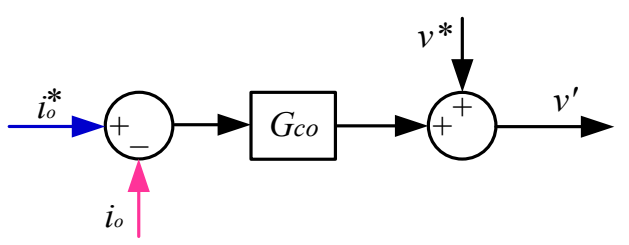

Fig. 8. AMS control blocks.

The AMS control block is shown in Fig. 8. The output current of each module forms the outer output current control loop. The key point is choosing the appropriate output current reference $i_{o}^{*}$.

This reference current is determined by the maximum current among all modules' output current, which is shown in Fig. 9. It can be easily understood that the module with maximum output current will automatically become the master module because the outer current loop will be eliminated. This module will work at voltage control mode to maintain the DC bus voltage. The remaining modules will follow the maximum current reference to sharing the power equally from the master module.

$$
i_{o}^{*}=\max \left\{\left|i_{o .1}\right|,\left|i_{o .2}\right|, \cdots,\left|i_{o . n}\right|\right\}
$$

Once the master module is plugged-out, the maximum current controller will automatically select another maximum current reference (also as the master module) to make the DC system work as normal. Similarly, if it has other modules plugged-in, they will obey the same rules.

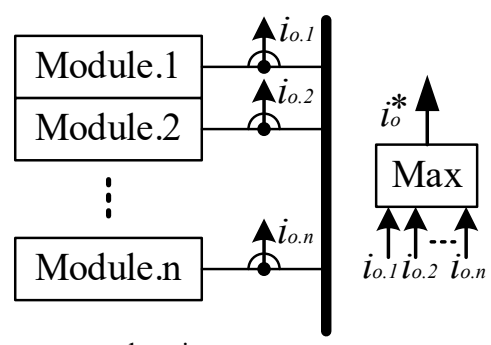

Fig. 9. Reference current choosing.

The controller shown in Fig. 8 is also a PI type controller, which can be written in equation (6).

$$
G_{c o}=G_{o m} \cdot\left(1+\frac{\omega_{z o}}{s}\right)
$$

Combining the above sections, the intact control block is shown in Fig. 10. The controller includes inner inductor current control, middle output voltage control, droop control and outer output current control. The first three control loop forms primary controller and outer output current control loop is secondary control.

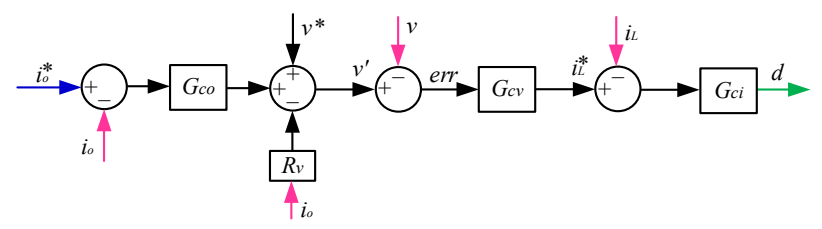

Fig. 10. Intact control blocks of converter. 


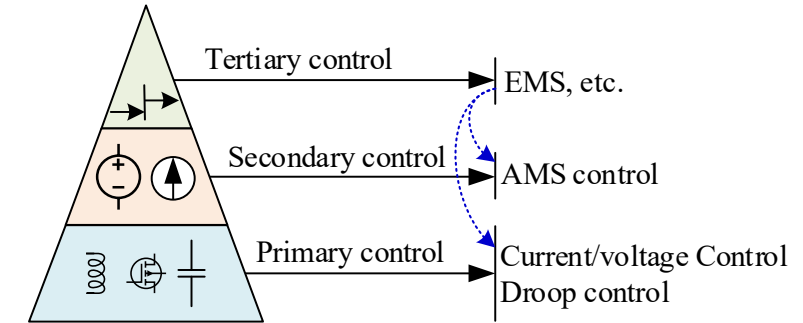

Fig. 11. Proposed control scheme in hierarchy.

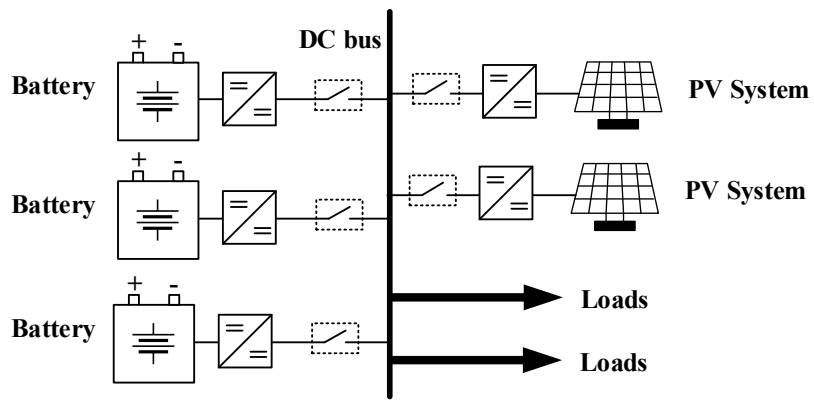

Fig. 12. Configuration of simulation system.

In practical, the power sources in a DC microgrid are formed by battery storage, utility grid, fuel cell or even electrical vehicle. Battery storage are mostly used to maintain the DC bus voltage due to its feasibility and convenience in bidirectional power flow. In this sense, it is always required to maintain the battery storage with longest lifespan otherwise it need to have a convenient and effective option to replace the used battery.

In the proposed control scheme, assuming the battery storage is used to maintain the DC bus voltage, multiple battery storage can be applied as they share identical output characteristic. Even one of the battery storages is about to reach the end of life-time, it can be easily plugged-out and replaced by a new one. For an isolated DC microgrids without any other voltage source, the droop coefficient can be set to zero so that the bus voltage can be always constant. If the DC system has other voltage sources, droop control can be applied on all voltage sources, communication in tertiary control may take in charge of the power and energy scheduling.

The parameters can be modified and control by tertiary communications through algorithms are output current reference $i_{o}^{*}$, droop coefficient $R_{v}$, and voltage reference $v^{*}$. However, the energy management algorithms to update those parameters are beyond the scope of this paper.

\section{Simulation Results}

The simulation diagram is shown in Fig. 12, which includes three battery banks, PV generations and loads. The parameters and values used in the simulation are listed in the Table I.

\section{A. Battery Current Sharing Test}

The results of battery current sharing test are shown from Fig. 13 to Fig. 15. Firstly, the droop coefficients of module 1, 2 and 3 are set as $0.5 \Omega, 2 \Omega$ and $4 \Omega$ respectively, and the cable resistance of each module are set same value $(1 \Omega)$. From 0 to $0.1 \mathrm{~s}$, it can be seen from Fig. 13 that the discharging (positive) current in each module is not same, which is within the expectation. The PV generation is plugged-in at $0.1 \mathrm{~s}$ and provides sufficient power to the load. Therefore, the
TABLE I. PARAMETERS AND VALUES USED IN THE SIMULATION.

\begin{tabular}{|c|c|l|}
\hline Parameters & Values & \multicolumn{1}{|c|}{ Explanations } \\
\hline$C$ & $470 \mathrm{uF}$ & capacitor \\
\hline$L$ & $1.2 \mathrm{mH}$ & Inductor \\
\hline$f_{s}$ & $10 \mathrm{kHz}$ & Switching frequency \\
\hline$R_{v}$ & $1 \sim 10 \Omega$ & Range of virtual resistance \\
\hline$R_{\text {line }}$ & $1 \sim 10 \Omega$ & Range of cable resistance \\
\hline$v_{g}$ & $150 \sim 170 \mathrm{~V}$ & Input source voltage \\
\hline$L o a d$ & $0 \sim 5 \mathrm{~kW}$ & Load range \\
\hline$f_{z i} / \omega_{z i}$ & $400 \mathrm{~Hz}$ & Inductor current controller frequency \\
\hline$f_{z v} / \omega_{z v}$ & $33.3 \mathrm{~Hz}$ & Voltage controller frequency \\
\hline$f_{z o} / \omega_{z o}$ & $10 \mathrm{~Hz}$ & Output current controller frequency \\
\hline$v_{b u s}$ & $380 \mathrm{~V}$ & DC bus voltage \\
\hline$P_{P V}$ & $5 \mathrm{~kW}$ & Power of PV generations \\
\hline
\end{tabular}

remaining power are injected to the battery storage automatically. The output current of each module goes into negative (charging) value. However, they are still not sharing equally and depend on the values of virtual resistance and cable resistance.

Secondly, the AMS controller is added to the control blocks. The same procedure is conducted, and results are shown in Fig. 14. From 0 to $0.1 \mathrm{~s}$, the discharging (positive) current of each module are equal. When the PV generation starts to power the microgrids, the charging current of each module are also same as predicted. Those results indicate the effectiveness of proposed AMS control.

Thirdly, taking the cable resistance into account, the cable resistance of module 1,2 and 3 are renewed as $1 \Omega, 4 \Omega$ and 6 $\Omega$, respectively. The same procedure is conducted again. From the Fig. 15, it can be seen that the current sharing between each module is still strictly same as predicted and follow the design. Those results also match the analysis of current sharing accuracy in MS control.
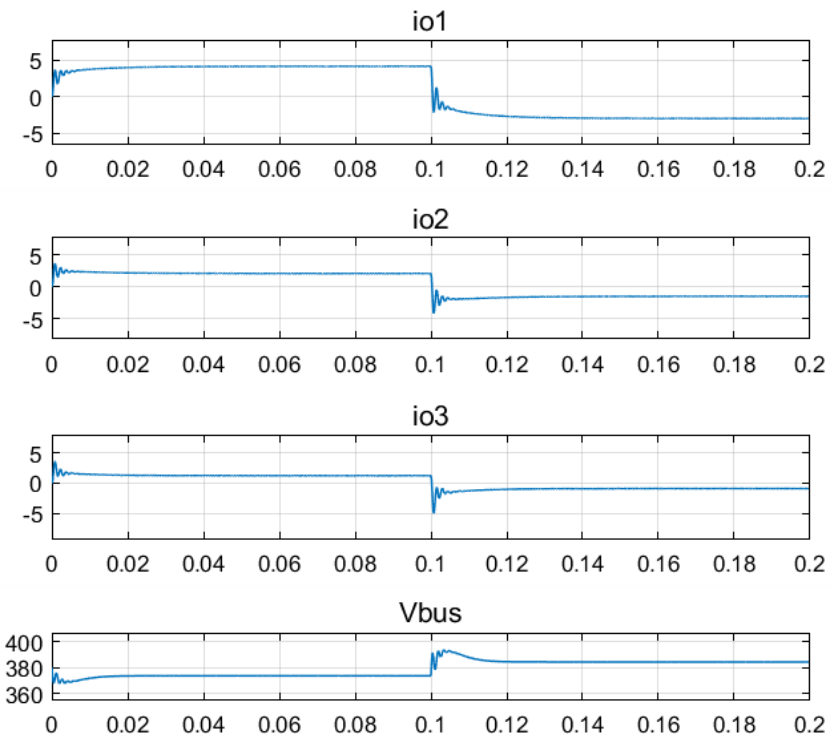

ffset $=0$

Fig. 13. Current sharing results without AMS and PV generation is pluggedin at $0.1 \mathrm{~s}$. 

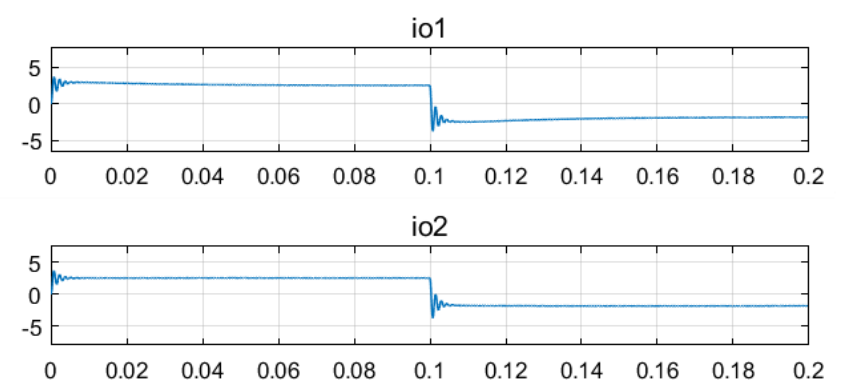

io3
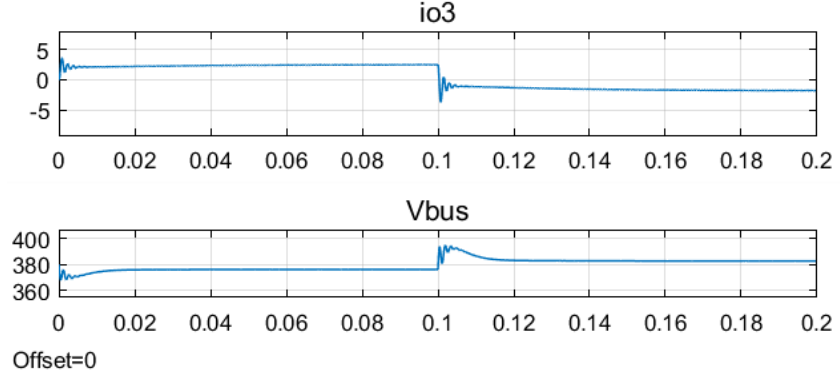

Fig. 14. Current sharing results with AMS and PV generation is plugged-in at $0.1 \mathrm{~s}$.
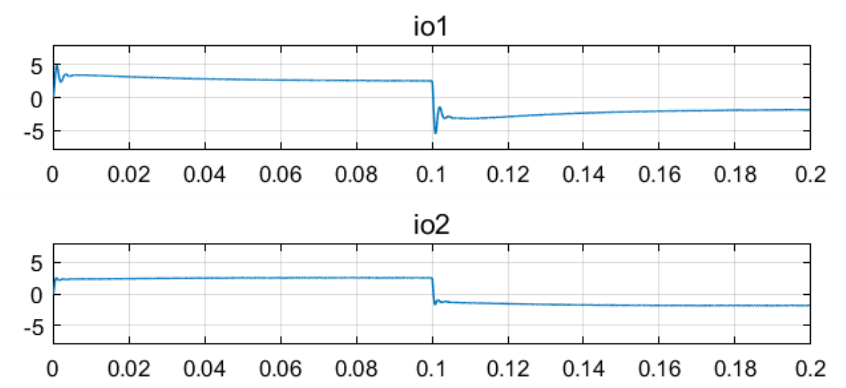

io3
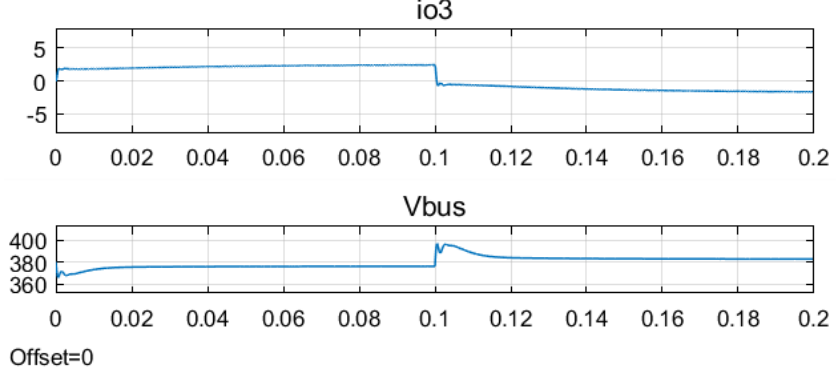

Fig. 15. Current sharing results with AMS, PV generation is plugged-in at $0.1 \mathrm{~s}$ and considering variation of cable resistance.

\section{B. Plug-and-play Test}

The P\&P performance test is shown in Fig. 16 and Fig. 17. First, the procedure that takes place between 0 and $0.2 \mathrm{~s}$ are same as before. The master module 1 is plugged-out at $0.2 \mathrm{~s}$, which can be seen from Fig. 16, the output current goes to zero. The DC bus voltage is still maintained even though the absence of master module. A new master is chosen to maintain the dc bus voltage. Module 2 and module 3 continue the even current sharing the surplus power from the PV generations.

Second, module 3 is not online in the beginning and then plugged-in at $0.2 \mathrm{~s}$, which is shown in Fig. 17. It can be seen that the final equal current sharing can still be achieved. This further proves the effectiveness of proposed multifunctional control in P\&P DC microgrids.
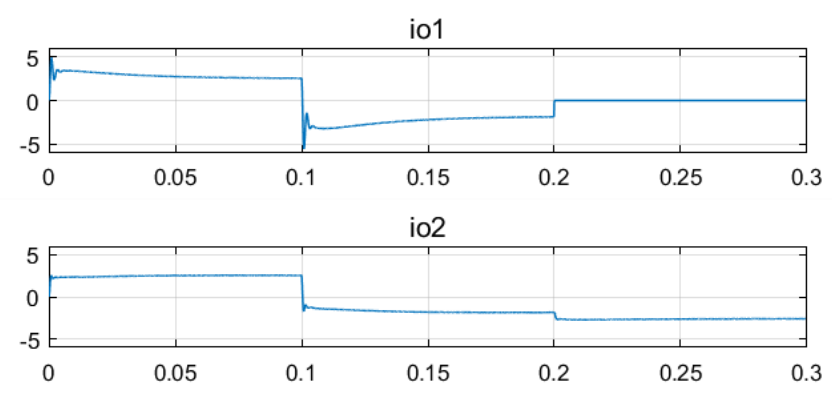

io3
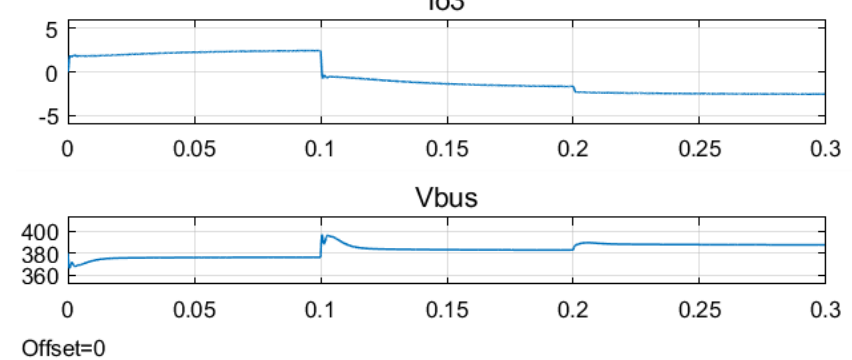

Fig. 16. Current sharing results with AMS, PV generation is plugged-in at $0.1 \mathrm{~s}$ and module 1 is plug-out at $0.2 \mathrm{~s}$.
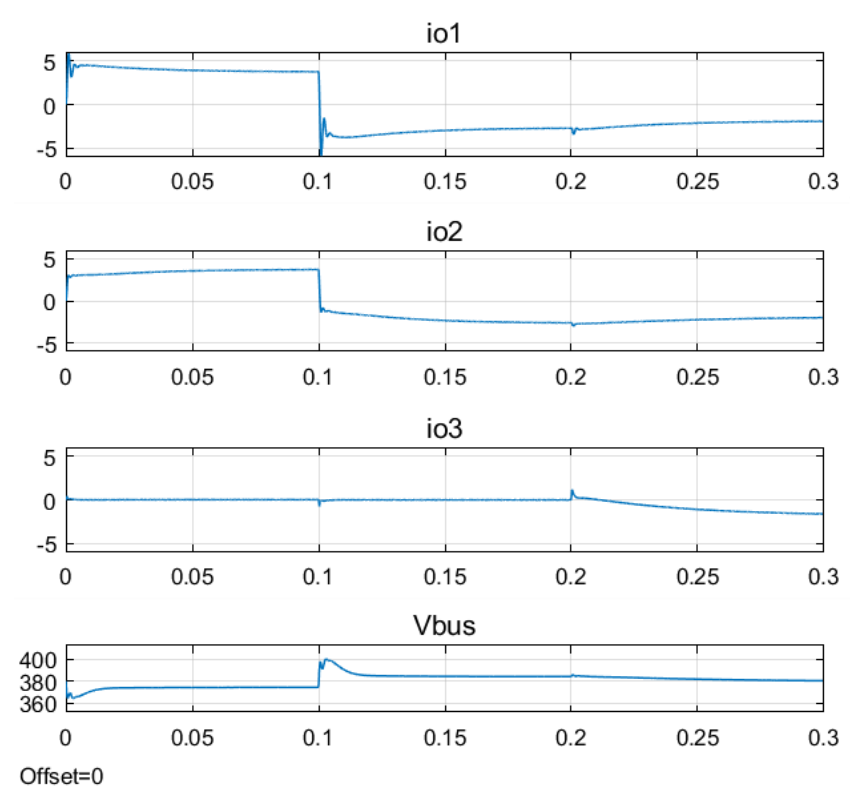

Fig. 17. Current sharing results with AMS, PV generation is plugged-in at $0.1 \mathrm{~s}$ and module 3 is plug-in at $0.2 \mathrm{~s}$.

\section{CONCLUSIONS}

This paper proposed a control scheme for modular plugand-play DC microgrids. In such control scheme, the battery storage can be operated with high flexibility and scalability in terms of plug-and-play DC microgrids. The proposed control scheme is ideal for battery energy management in a hierarchically controlled DC microgrids. In this work, the communication is not implemented and in fact, the current in each battery module is not merely average current sharing. A simple gain can be added to output reference to adjust the current sharing if communication is added too. This work will be further studied in the future.

\section{REFERENCES}

[1] F. Li, Z. Lin, Z. Qian, J. Wu and W. Jiang, "A Dual-Window DC Bus Interacting Method for DC Microgrids Hierarchical Control Scheme," in IEEE Transactions on Sustainable Energy. 
[2] X. Yu, F. Wang and A. Q. Huang, "Power management strategy for plug and play DC microgrid," 2012 3rd IEEE PES Innovative Smart Grid Technologies Europe (ISGT Europe), Berlin, 2012, pp. 1-7.

[3] J. M. Guerrero, J. C. Vasquez, J. Matas, L. G. de Vicuna and M. Castilla, "Hierarchical Control of Droop-Controlled AC and DC Microgrids-A General Approach Toward Standardization," in IEEE Transactions on Industrial Electronics, vol. 58, no. 1, pp. 158-172, Jan. 2011

[4] X. Lu, J. M. Guerrero, K. Sun and J. C. Vasquez, "An Improved Droop Control Method for DC Microgrids Based on Low Bandwidth Communication With DC Bus Voltage Restoration and Enhanced Current Sharing Accuracy," in IEEE Transactions on Power Electronics, vol. 29, no. 4, pp. 1800-1812, April 2014.

[5] F. Li, Z. Lin, W. Cao, A. Chen and J. Wu, "A Low-pass Filter Method to Suppress the Voltage Variations Caused by Introducing Droop Control in DC Microgrids," 2018 IEEE Energy Conversion Congress and Exposition (ECCE), Portland, OR, 2018, pp. 1151-1155.

[6] F. Li, Z. Lin, J. Wu and W. Li, "Virtual Negative Cable Resistance for Power Sharing Accuracy Enhancement in DC Microgrids," 2019 IEEE 28th International Symposium on Industrial Electronics (ISIE), Vancouver, BC, Canada, 2019, pp. 2539-2544.

[7] Z. Jin and J. M. Guerreros, "Two-degree-of-freedom admittance-type droop control for plug-and-play DC microgrid," 2018 IEEE Applied Power Electronics Conference and Exposition (APEC), San Antonio, TX, 2018, pp. 3326-3332.
[8] M. Tucci, S. Riverso and G. Ferrari-Trecate, "Line-Independent Plugand-Play Controllers for Voltage Stabilization in DC Microgrids," in IEEE Transactions on Control Systems Technology, vol. 26, no. 3, pp. 1115-1123, May 2018.

[9] D. Xu, A. Xu, C. Yang and P. Shi, "Uniform State-of-Charge Control Strategy for Plug-and-Play Electric Vehicle in Super-UPS," in IEEE Transactions on Transportation Electrification, vol. 5, no. 4, pp. 11451154, Dec. 2019.

[10] J. Rajagopalan, K. Xing, Y. Guo, F. C. Lee and B. Manners, "Modeling and dynamic analysis of paralleled DC/DC converters with masterslave current sharing control," Proceedings of Applied Power Electronics Conference. APEC '96, San Jose, CA, USA, 1996, pp. 678684 vol.2.

[11] Y. Panov, J. Rajagopalan and F. C. Lee, "Analysis and design of N paralleled DC-DC converters with master-slave current-sharing control," Proceedings of APEC 97 - Applied Power Electronics Conference, Atlanta, GA, USA, 1997, pp. 436-442 vol.1.

[12] R. Ahmadi and M. Ferdowsi, "Controller design method for a cascaded converter system comprised of two DC-DC converters considering the effects of mutual interactions," 2012 Twenty-Seventh Annual IEEE Applied Power Electronics Conference and Exposition (APEC), Orlando, FL, 2012, pp. 1838-1844.

[13] R. W. Erickson and D. Maksimović, "Current Programmed Control," in Fundamentals of Power Electronics, Boston, MA: Springer US, 2001, pp. 439-487. 